\title{
A Novel Diversity Receiver Structure for Severe Fading and Frequency Offset Conditions
}

\author{
Bojan DIMITRIJEVIC ${ }^{1}$, Vladimir KRSTIC ${ }^{2}$, Bojana NIKOLIC ${ }^{3}$ \\ ${ }^{1}$ Dept. of Telecommunications, University of Niš, A. Medvedeva 14, 18000 Niš, Serbia \\ ${ }^{2}$ Institute "Mihajlo Pupin", University of Belgrade, Volgina 15, 11060 Belgrade, Serbia \\ ${ }^{3}$ Dept. of Theoretical Electrical Engineering, University of Niš, A. Medvedeva 14, 18000 Niš, Serbia \\ bojan.dimitrijevic@elfak.ni.ac.rs, vladimir.krstic@pupin.rs, bojana.nikolic@elfak.ni.ac.rs
}

\begin{abstract}
This paper presents a novel diversity receiver of MPSK signal in fading channel in the presence of the carrier frequency offset. As a part of this receiver, a new algorithm for the estimation of the combining coefficients (ECC algorithm) is introduced. Having in mind that the QPSK modulation is one of the most used modulation formats in many wireless communication standards (LTE, WiFi, WiMax), the performance of the proposed receiver is analyzed in more detail for the QPSK modulation. In the presence of Rayleigh fading, representing the most severe fading condition, this algorithm shows significantly better performance comparing to the same receiver structure that uses conventional constant modulus algorithm (CMA1 or CMA2). The proposed diversity receiver structure with ECC algorithm operates within a wide carrier frequency offset range with a very small variation of the performance. For this reason, it can be applied in $4 G$ mobile communication systems.
\end{abstract}

\section{Keywords}

Fading, constant modulus algorithm, adaptive filtering, frequency offset, diversity receiver

\section{Introduction}

A presence of fading in the communication channel may significantly degrade performance of the wireless transmission. For this reason, a diversity receiver is often used to improve the transmission quality. There are three basic combining concepts available in the literature: maximum ratio combining (MRC) [1], [2], equal-gain combining (EGC) [3-7] or selection combining (SC) [1], [2] technique. In practice, the combining is often realized using the constant modulus algorithm (CMA) [8]. On the other hand, the important drawback of the CMA is its relatively slow convergence [9]. In order to improve some of the characteristics of the conventional CMA, various modifications of this algorithm have been made. Also, the convergence characteristics of the existing low complexity stochastic gradient (SG) type of algorithms have been improved in order to ensure good tracking of channels and interferences in the wireless networks characterized by nonstationary environments.

The normalized CMA (NCMA), presented in [10], employs the normalized step size which is proportional to the required one to achieve the desired modulus with the current data vector. The convergence behavior and the implementation cost of the normalized CMA algorithm are analogue to that of the normalized LMS algorithm. In [11], the modified CMA algorithm, which performs blind equalization and carrier phase recovery, is extrapolated to the fourth order and applied to the decision feedback equalizer. In comparison with the MCMA, the use of high order statistics reduces considerably the effect of Gaussian noise and leads to the lower residual MSE of the equalizer. In [12], the CMA algorithm with a variable step-size, which is based on the autocorrelation functions between $\mathrm{N}$ previous error functions, is proposed and verified using IEEE 802.16-2004 SC physical layer computer simulations. In [13], [14] and [15], the low-complexity variable step size mechanisms are proposed for SG algorithms. The minimum variance [13] and code-constrained constant modulus [15] SG algorithms are used for multiple access interference and intersymbol interference suppression in CDMA systems, and in [14], the constrained constant modulus SG algorithms are proposed for adaptive beamforming.

Besides the fading, the carrier frequency offset is also a very frequent disturbing effect that appears in wireless transmission channel. Receivers that show good performance in wide range of frequency offsets have been proposed in [16] and [17] for BPSK and MDPSK modulation formats, respectively.

The M-ary phase shift keying (MPSK) diversity receiver presented in this paper is suitable for operation in fading channel and in the presence of carrier frequency offset. It is known that CMA is resistant to the carrier frequency offset (its performance is negligibly changed in the presence of frequency offset). For this reason, CMA has been considered to be used for combining coefficient adjustment. However, its relatively slow convergence is quite a drawback in the receiver that operates in fading condi- 
tions. As a solution to this problem, a new algorithm for combining coefficient adjustment is introduced in this paper. In comparison to CMA, this new algorithm provides better performance in the above mentioned propagation conditions and it is also resistant to the carrier frequency offset. Considering good characteristics of the receiver described in [16] in the presence of the carrier frequency offset and its low complexity, the recursive filter of unitary length with remodulation [16] is chosen to be used for the detection process. In this way, the proposed MPSK receiver significantly improves system performance in the presence of fading and still retains a desirable feature to operate within a wider carrier frequency offset range with a very small variation of the performance.

The paper is organized as follows. Section 2 describes the system model, and the selected numerical results are given in Sec. 3. Section 4 concludes the paper with some final remarks.

\section{System Model}

A block diagram of the proposed MPSK signal receiver is presented in Fig. 1. For diversity receiver with $N_{a}$ antennas, the input signal at each antenna can be presented as:

$$
r_{i}(t)=s_{i}(t)+n_{i}(t), i=0,1, \ldots, N_{a}-1,
$$

where $n_{i}(t)$ is the white Gaussian noise, and $s_{i}(t)$ is the MPSK signal with a rectangular symbol pulse shape:

$$
s_{i}(t)=\exp \left[j \frac{2 \pi}{M} d\left(t-\tau_{i}\right)\right] \exp \left[j \hat{\omega}_{c}\left(t-\tau_{i}\right)\right] .
$$

The number of modulation levels is $M$. In (2), the symbol $d(t)$ takes one of the following values:

$$
d(t) \in\{0,1 \ldots, M-1\}, k T_{s} \leq t<(k+1) T_{s}, k=0,1,2 \ldots
$$

$T_{s}$ is the symbol interval, $\tau_{i}$ is the delay at the $i$-th path, $\hat{\omega}_{c}=\omega_{c}+\Delta \omega$ is the carrier frequency at the input of the receiver, $\omega_{c}$ is the locally generated fixed reference carrier frequency and $\Delta \omega$ is the frequency offset.

The complex-valued baseband signal at the input of the combiner is obtained by multiplying the input signal in each diversity branch by the fixed frequency reference carrier and leading the resulting signal through the integrating and dump circuit. This signal can be expressed as:

$$
X_{i}(k)=x_{I, i}(k)+j x_{Q, i}(k)
$$

where its in-phase $x_{I, i}(k)$ and quadrature $x_{Q, i}(k)$ components are given by:

$$
\begin{aligned}
& x_{I, i}(k)=\int_{k T_{s}}^{(k+1) T_{s}} r_{i}(t) \cos \left(\omega_{c} t\right) d t \\
& x_{Q, i}(k)=\int_{k T_{s}}^{(k+1) T_{s}} r_{i}(t) \sin \left(\omega_{c} t\right) d t .
\end{aligned}
$$

$k$ denotes the discrete time corresponding to the output of the integrate and dump circuit.

The signal at the output of the combiner is:

$$
Z(k)=\sum_{i=0}^{N_{a}-1} X_{i}(k) V_{i}(k)
$$

where $N_{a}$ is the number of diversity branches, $V_{i}(k)$ is the weight of $i$-th branch.

In this paper a new algorithm for estimation of combining coefficients (ECC) in diversity combiner is proposed. The performance of such a combiner converges to the performance of MRC combining. The optimal values of MRC weight coefficients can be presented as [18]:

$$
V_{i}(k)=C_{S i}^{*}(k)
$$

where $C_{S i}(k)$ is a complex baseband channel coefficient corresponding to the $i$-th diversity branch, and $(\cdot)^{*}$ is the complex conjugate of $(\cdot)$.

In order to explain the physical meaning of transformations that are introduced in the proposed algorithm, let us present the received signal $X_{i}(k)$ in the $i$-th diversity branch as:

$$
\begin{aligned}
X_{i}(k) & =S(k) C_{S i}(k)+n_{i}(k) \\
& =A_{S}(k) \cdot \exp \left[j \frac{\pi}{2} d(k)\right] C_{S i}(k)+n_{i}(k)
\end{aligned}
$$

where $A_{S}(k)$ is the amplitude of the useful signal, $d(k)$ is discrete form of $d(t)$ from (3). $n_{i}(k)$ is additive White Gaussian Noise (AWGN) with variance $\sigma_{i}{ }^{2}$.

Substituting (8) in (6) one can obtain the signal at the output of the combiner:

$$
Z(k)=A_{S}(k) \exp \left[j \frac{\pi}{2} d(k)\right] \underbrace{\sum_{i=1}^{N_{a}} C_{S i}(k) V_{i}(k)}_{C_{e}(k)}+n_{e}(k)
$$

where $n_{e}(k)=\sum_{i=1}^{N_{a}} V_{i}(k) n_{i}(k)$ is AWGN with variance $\sigma_{e}{ }^{2}$.

The variable $C_{e}(k)$, introduced in (9), is assumed to be slowly varying and can be written in a form:

$$
C_{e}(k)=\sum_{i=1}^{N_{a}} C_{S i}(k) V_{i}(k)=A_{e}(k) \exp \left[j \phi_{e}(k)\right] .
$$

Now expression (9) becomes:

$$
Z(k)=A_{S}(k) A_{e}(k) \exp \left[j \frac{\pi}{2} d(k)\right] \exp \left[j \phi_{e}(k)\right]+n_{e}(k) \cdot(11)
$$

Considering (7), it is necessary to determine channel coefficient $C_{S i}(k)$. In the first term of the summation (8), besides a desired channel coefficient, there is also a fast varying value $\mathrm{e}^{j d(k) \pi / 2}$. The same value exists also in (11). In order to eliminate the fast-varying value, a new variable is introduced: 


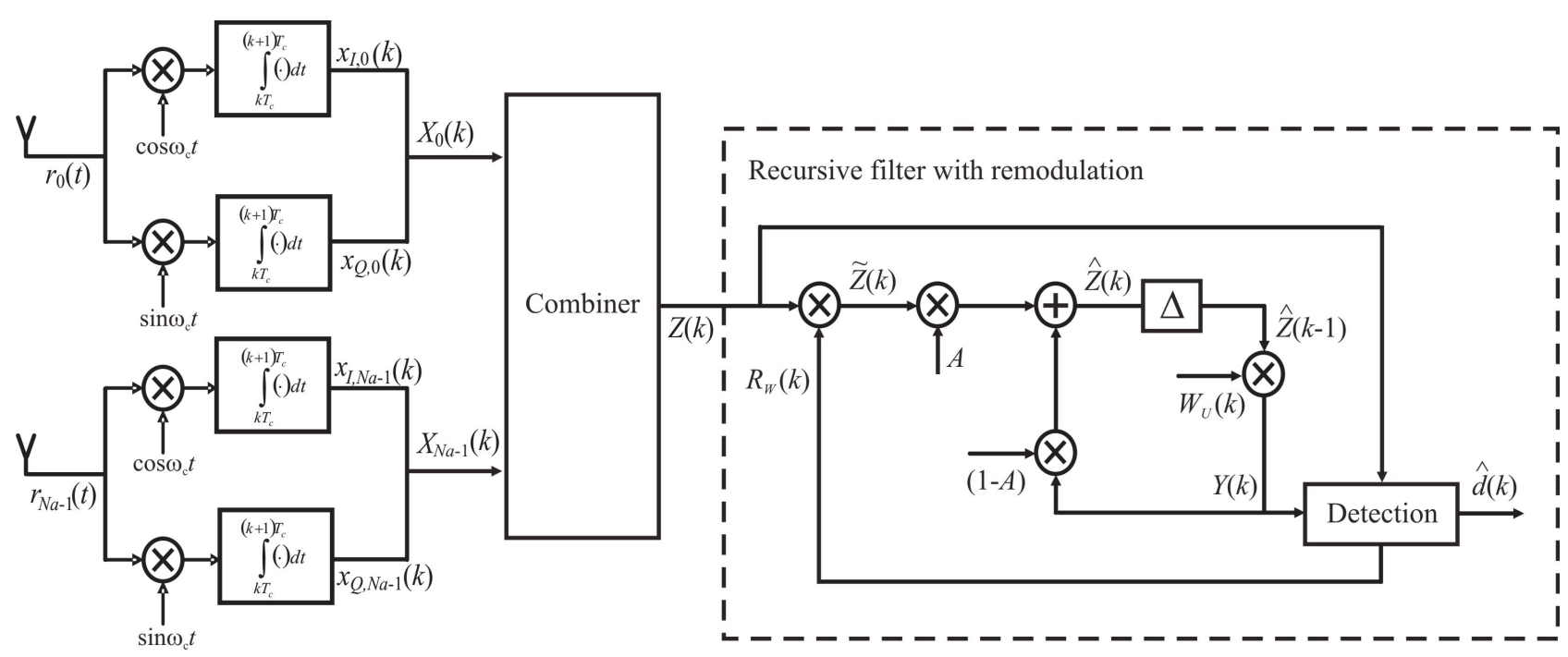

Fig. 1. Block diagram of the proposed receiver.

$$
\tilde{V}_{i}(k)=\frac{Z(k)}{|Z(k)|} X_{i}^{*}(k)
$$

Substituting (11) and (8) in (12), it is obtained:

$$
\tilde{V}_{i}(k)=C_{S i}^{*}(k) A_{s}(k) \exp \left[j \phi_{e}(k)\right]+n_{e}^{\prime}(k) .
$$

It can be seen from (13) that the variable $\tilde{V}_{i}(k)$ could be the solution for estimation of combining coefficients in diversity combiner, since it is proportional to the optimal value of MRC weight coefficients $\left(\tilde{V}_{i}(k) \sim C_{S i}{ }^{*}(k)\right)$ in the presence of noise. The variable $\tilde{V}_{i}(k)$ can be considered as an instantaneous estimated value of the coefficient in the $i$ th diversity branch. Thus, its average value can be obtained applying low-pass filter (LPF). In that case, signal at the output of LPF has the form:

$$
V_{i}(k)=(1-\alpha) V_{i}(k-1)+\alpha \tilde{V}_{i}(k)
$$

where $\alpha$ is the parameter $(0<\alpha<1)$ that represents the LPF smoothing factor.

Substituting (12) in (14), the ECC algorithm for estimation of combining coefficients in diversity combiner is obtained and it is described by the relation:

$$
V_{i}(k)=(1-\alpha) V_{i}(k-1)+\alpha \frac{Z(k)}{|Z(k)|} X_{i}^{*}(k) .
$$

Results obtained using this algorithm will be compared with the ones obtained using CMA1 and CMA2 algorithms [19]. In the case of using CMA1 algorithm, $V_{i}(k)$ can be written as [19]:

$$
\begin{aligned}
& V_{i}(k)=V_{i}(k-1)+ \\
& \mu_{V 1}\left(\frac{1}{|Z(k-1)|}-1\right) Z(k-1) X_{i}^{*}(k-1)
\end{aligned}
$$

and in the case of CMA2 algorithm, the weight of the $i$-th branch is described by [19]:

$$
\begin{aligned}
& V_{i}(k)=V_{i}(k-1)+ \\
& \mu_{V 2}\left(1-|Z(k-1)|^{2}\right) Z(k-1) X_{i}^{*}(k-1)
\end{aligned}
$$

where $\mu_{V 1}$ and $\mu_{V 2}$ are the adaptation factors.

Considering good performance of the receiver described in [16] in the presence of carrier frequency offset, the recursive filter with remodulation is used for the detection process in the receiver that is proposed here. Since the influence of the recursive filter length on the error probability is negligible [16], we propose to use the transversal filter of unitary length in this block, due to its simplicity. In that case the signal $\hat{Z}(k)$ may be written as:

$$
\hat{Z}(k)=A \tilde{Z}(k)+(1-A) W_{U}(k) \hat{Z}(k-1)
$$

where $A$ denotes the introduced constant parameter $(A \leq 1)$. The value $(1-A)$ defines a part of the output signal that is returned to the input. We get $\hat{Z}(k)$ after the remodulation:

$$
\tilde{Z}(k)=R_{W}(k) Z(k)
$$

where $R_{W}(k)$ is the remodulation weight.

The adjustment of the weights $W_{U}(k)$ is performed by the normalized LMS algorithm [20], [21]:

$$
W_{U}(k+1)=W_{U}(k)+\frac{\mu_{U} E(k) \hat{Z}^{*}(k-1)}{\overline{|\hat{Z}(k)|^{2}}}
$$

where $\mu_{U}$ is the adaptation factor.

The error signal is obtained as:

$$
E(k)=R_{W}(k) Z(k)-Y(k)=\tilde{Z}(k)-Y(k)
$$


where

$$
Y(k)=W_{U}(k) \hat{Z}(k-1) .
$$

The detected symbol is obtained by the following minimization:

$$
\hat{d}(k)=\underset{r \in\{0,1, \ldots, M-1\}}{\arg \min }\left|\exp \left[j \frac{2 \pi r}{M}\right] Z(k)-Y(k)\right|^{2} .
$$

The remodulation weight is:

$$
R_{W}(k)=\exp \left[j \frac{2 \pi \hat{d}(k)}{M}\right] .
$$

\section{Numerical Results}

The results shown in the following figures are obtained by the Monte-Carlo simulation with 10 million steps. The application is written in $\mathrm{C}^{++}$and executed on an Intel ${ }^{\circledR}$ Core $^{\mathrm{TM}}$ i5-3470 CPU @ 3.20 GHz. The simulation time is 30 minutes per curve. Carrier frequency is $f_{c}=2.44 \mathrm{GHz}$ and bit rate is $V_{t}=T_{b}^{-1}=100 \mathrm{kbs}^{-1}$. The detection of MPSK signals in non-selective Rayleigh and Rician fading channel or AWGN channel in the presence of carrier frequency offset is considered. The Rayleigh fading consists only of the diffuse component that is modeled by using 10 equal power virtual sources of the transmitted signal. These sources are randomly placed around the mobile unit. In case of Rician fading, besides the diffuse, there is the direct component from another source. The direct to the diffuse component power ratio is given by parameter $K$. A diversity system with four branches $\left(N_{a}=4\right)$ is applied at the reception. The optimized system parameters are: $A=0.2, \mu_{U}=0.05, \alpha=0.05, \mu_{V I}=10^{-3}$ and $\mu_{V 2}=5 \times 10^{-3}$.

Table 1 shows the comparative values of the error probability for the three observed combiner coefficients adjustment algorithms (ECC, CMA1, and CMA2) in the Rayleigh fading channel. Different modulation formats BPSK $(M=2)$, QPSK $(M=4)$ and 8PSK and different velocities of the mobile unit are considered. For the considered modulation formats (BPSK, QPSK and 8PSK) different signal to noise ratio (SNR) values are chosen $(4 \mathrm{~dB}, 7 \mathrm{~dB}$, and $10 \mathrm{~dB})$ in order to achieve a similar error probability.

\begin{tabular}{|c|c|c|c|c|}
\hline $\begin{array}{c}\text { Velocity } \\
{\left[\mathrm{km}^{-1}\right]}\end{array}$ & $\begin{array}{c}\text { Modulation } \\
\text { format }\end{array}$ & ECC & CMA1 & CMA2 \\
\hline \multirow{3}{*}{0} & BPSK & 0.000011 & 0.000013 & 0.000012 \\
\cline { 2 - 5 } & QPSK & 0.000015 & 0.000022 & 0.000021 \\
\cline { 2 - 5 } & 8PSK & 0.0009186 & 0.0008950 & 0.0009219 \\
\hline \multirow{3}{*}{50} & BPSK & 0.0009287 & 0.0078234 & 0.0026852 \\
\cline { 2 - 5 } & QPSK & 0.0019331 & 0.0204117 & 0.0051332 \\
\cline { 2 - 5 } & 8PSK & 0.0178139 & 0.115171 & 0.0385318 \\
\hline \multirow{3}{*}{100} & BPSK & 0.0010801 & 0.0057136 & 0.0022814 \\
\cline { 2 - 5 } & QPSK & 0.0021501 & 0.0139157 & 0.0044921 \\
\cline { 2 - 5 } & 8PSK & 0.0169559 & 0.0822456 & 0.0357827 \\
\hline
\end{tabular}

Tab. 1. Error probability for different modulation formats and different values of mobile unit velocity in AWGN and Rayleigh fading channel.
The value of the normalized frequency offset is set to $\Delta f \times T_{s}=0.06$. Table shows that for the mobile unit velocity $V=0 \mathrm{kmh}^{-1}$ all three combiner coefficients adjustment algorithms provide similar results, regardless of the modulation format. It can also be concluded that for moving mobile unit, the ECC algorithm always produces the best results, regardless of the modulation format and velocity.

Table 2 refers to the Rician fading channel. The ratio of direct and diffuse component power, in this case, is $K=3 \mathrm{~dB}$. Other parameters retain the same numerical values as for Table 1. Again, the best results are achieved by the ECC algorithm, regardless of the modulation format and the mobile unit velocity.

\begin{tabular}{|c|c|c|c|c|}
\hline $\begin{array}{c}\text { Velocity } \\
{\left[\mathrm{kmh}^{-1}\right]}\end{array}$ & $\begin{array}{c}\text { Modulation } \\
\text { format }\end{array}$ & ECC & CMA1 & CMA2 \\
\hline \multirow{3}{*}{0} & BPSK & 0.000012 & 0.000013 & 0.000012 \\
\cline { 2 - 5 } & QPSK & 0.0000151 & 0.000015 & 0.000015 \\
\cline { 2 - 5 } & 8 PSK & 0.0007672 & 0.00072009 & 0.0007503 \\
\hline \multirow{3}{*}{50} & BPSK & 0.00023218 & 0.00042398 & 0.00025237 \\
\cline { 2 - 5 } & QPSK & 0.00037855 & 0.00118614 & 0.00072177 \\
\cline { 2 - 5 } & 8PSK & 0.00499356 & 0.0271516 & 0.00785038 \\
\hline \multirow{3}{*}{100} & BPSK & 0.00019180 & 0.00053502 & 0.00024227 \\
\cline { 2 - 5 } & QPSK & 0.00033312 & 0.00256912 & 0.00057540 \\
\cline { 2 - 5 } & 8PSK & 0.00533678 & 0.0314284 & 0.00769559 \\
\hline
\end{tabular}

Tab. 2. Error probability for different modulation formats and different values of mobile unit velocity in AWGN and Rice fading channel.

Bearing in mind that the QPSK modulation is one of the most used modulation formats in many wireless communication standards (LTE, WiFi, WiMax), the performance of the proposed receiver will be analyzed in the remaining of the paper only for QPSK modulation. Also, the analysis in the remainder of the paper will be performed in the Rayleigh fading channel, because it represents the case of the most severe fading condition.

The dependence of error probability on the SNR is shown for QPSK modulation scheme in Fig. 2. The value of normalized frequency offset is set to $\Delta f \times T_{s}=0.05$. Curves denoted as Group $\boldsymbol{a}$ correspond to the transmission over AWGN channel. In that case all three previously mentioned algorithms for combining coefficient adjustment (the one that is proposed here, CMA1 and CMA2) give approximately the same results, regardless of SNR value. Curves denoted as Group $\boldsymbol{b}$ correspond to the case of transmission over Rayleigh fading channel. For all SNR values the receiver with combiner based on the ECC algorithm shows the best results. The worst results are obtained for the receiver with combiner based on CMA1. With the increase of SNR, the difference in performance for those two border cases becomes more emphasized.

In Fig. 3 the dependence of error probability on the carrier frequency offset, for QPSK modulation scheme, is presented for the case of Rayleigh fading channel. The velocity of the mobile unit is $V=50 \mathrm{kmh}^{-1}$. The value of SNR is set to be: $S N R=8 \mathrm{~dB}$. One can notice that in these operating conditions diversity receiver that uses the ECC algorithm for combining coefficient adjustment gives the best results. 


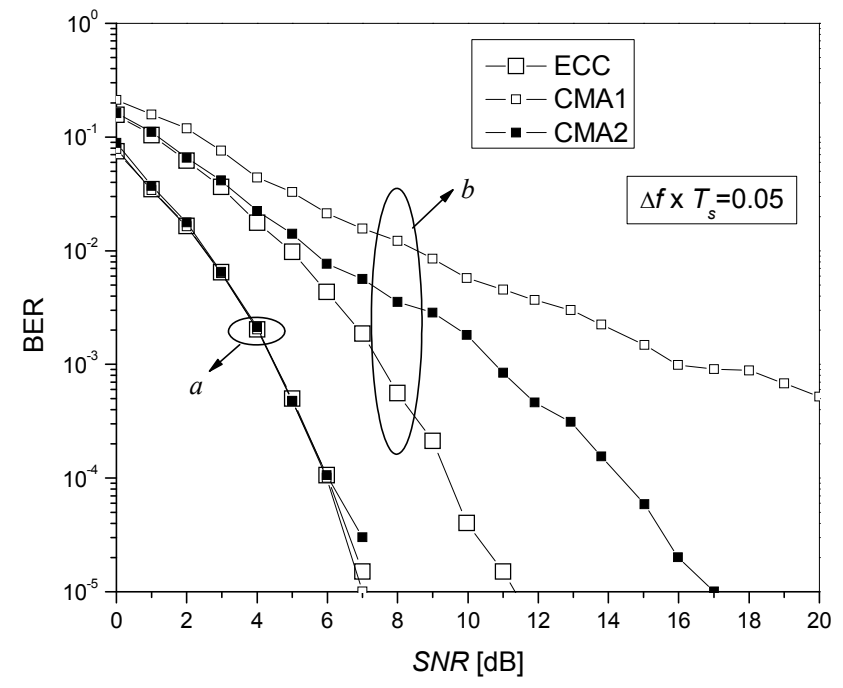

Fig. 2. Error probability as a function of signal to noise ratio: $\boldsymbol{a}$ - AWGN channel, $\boldsymbol{b}$ - Rayleigh fading channel.

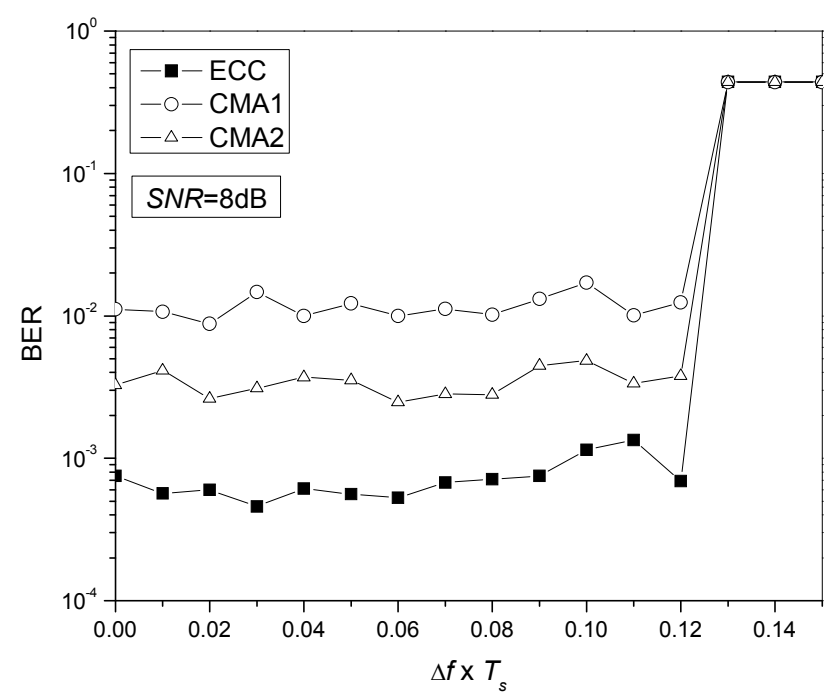

Fig. 3. Error probability as a function of carrier frequency offset in Rayleigh fading channel.

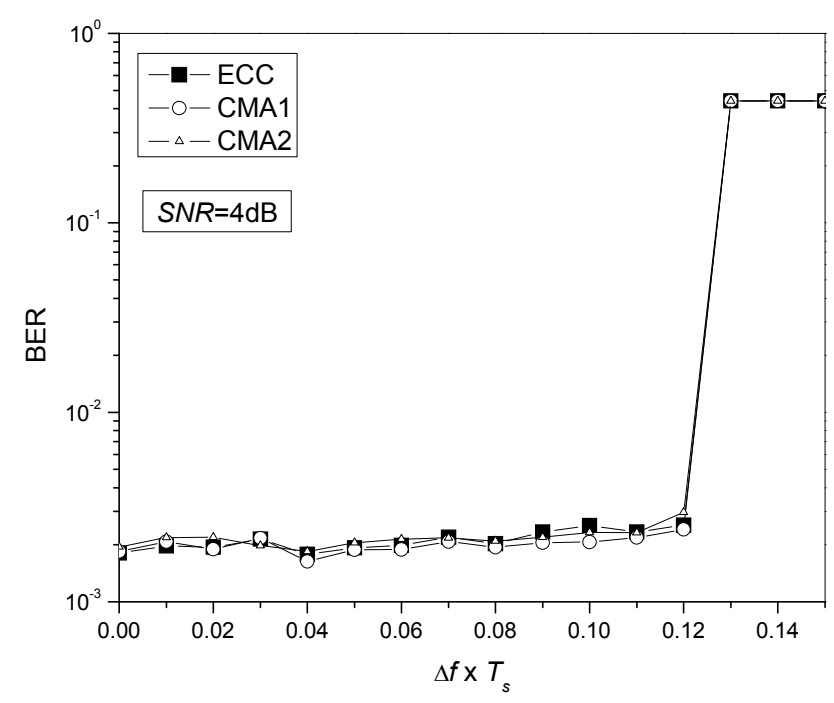

Fig. 4. Error probability as a function of carrier frequency offset in AWGN channel.
Also, it can be seen that for all three algorithms the frequency offset range, in which receiver performance shows only small variations, is approximately the same.

Fig. 4 illustrates the performance of the proposed diversity receiver in AWGN channel (the velocity of the mobile unit is $V=0 \mathrm{kmh}^{-1}$ ) for QPSK modulation scheme. The value of SNR is set to be: $S N R=4 \mathrm{~dB}$. In this case, all three considered algorithms for combining coefficient adjustment (ECC, CMA1 and CMA2) show similar results in wide range of carrier frequency offset values.

\section{Conclusion}

This paper proposes the MPSK diversity receiver for operation in fading channel in the presence of carrier frequency offset. The receiver performance is analyzed in more detail for the QPSK modulation, as it is one of the most widely used modulation formats in wireless communications. The proposed receiver uses the ECC algorithm for the adjustment of the combining coefficients and in the above mentioned propagation conditions shows significantly better performance in comparison with the same receiver structure that uses conventional constant modulus algorithm (CMA1 or CMA2).

It is also shown that the proposed diversity receiver can operate within a wide carrier frequency offset range with a very small variation of the performance using either the ECC algorithm or the conventional constant modulus algorithm.

\section{Acknowledgments}

This work was supported in part by the Serbian Ministry of Education and Science within the project "Research and development of robust systems for data transmission and its application in corporative networks", TR32037.

The authors thank the anonymous reviewers for their valuable suggestions and comments.

\section{References}

[1] SIMON, M. K., ALOUINI, M. S. Digital Communication over Fading Channels: A Unified Approach to Performance Analysis. New York (USA): Wiley, 2005. ISBN: 0-471-64953-8

[2] PROAKIS, J. G. Digital Communications. $4^{\text {th }}$ ed., New York (USA): McGraw-Hill, 2001. ISBN: 0072321113.

[3] KWOK, Y. -K. R., LAU, V. K. N. Wireless Internet and Mobile Computing: Interoperability and Performance. Hoboken (New Jersey, USA): John Wiley \& Sons, Inc., 2007. ISBN: 9780471679684

[4] PATTERH, M. S., KAMAL, T. S., SOHI, B. S. BER performance of MQAM with L-branch MRC diversity reception over correlated 
Nakagami-m fading channels. Wireless Communications and Mobile Computing, 2003, vol. 3, no. 3, p. 397-406. DOI: $10.1002 / \mathrm{wcm} .93$

[5] NIKOLIC, B. Z., DJORDJEVIC, G. T., KARAGIANNIDIS, G. K. On the effect of imperfect cophasing in MRC and EGC receivers over correlated Weibull fading. Wireless Personal Communications, 2012, vol. 62, no. 1, p. 31-39. DOI: 10.1007/s11277-010-0036-2

[6] NAJIB, M. A., PRABHU, V. K. Analysis of equal-gain diversity with partially coherent fading signals. IEEE Transactions on Vehicular Technology, 2000, vol. 49, no. 3, p. 783-791. DOI: 10.1109/25.845098

[7] SAGIAS, N. C., KARAGIANNIDIS, G. K. Effects of carrier phase error on EGC receivers in correlated Nakagami-m fading. IEEE Communications Letters, 2005, vol. 9, no. 7, p. 580-582. DOI: 10.1109/LCOMM.2005.07006

[8] TREICHLER, J. R., AGEE, B. G. A new approach to multipath correction of constant modulus signals. IEEE Transactions on Acoustics, Speech, and Signal Processing, 1983, vol. ASSP-28, p. 459-472. DOI: 10.1109/TASSP.1983.1164062

[9] GODARD, D. N. Self recovering equalization and carrier tracking in two dimensional data communication systems. IEEE Transactions on Communications, 1980, vol. 28, no. 11, p. 1867-1875. DOI: 10.1109/TCOM.1980.1094608

[10] JONES, D. L. A normalized constant modulus algorithm. In Conference Record of the 29th Asilomar Conference on Signals, Systems and Computers. Pacific Grove (USA), 1995, vol. 1, p. 694-697. DOI: 10.1109/ACSSC.1995.540639

[11] CHAHED, I., BELZILE, J., KOUKI, A. B. Blind decision feedback equalizer based on high order MCMA. In Proceedings of the 18th Canadian Conference on Electrical and Computer Engineering. Niagara Falls (Canada), 2004, vol. 4, p. 2111-2114. DOI: $10.1109 /$ CCECE.2004.1347658

[12] DEMIR, M. A., OZEN, A. A novel variable step size adjustment method based on autocorrelation of error signal for the constant modulus blind equalization algorithm. In Proceedings of the International Conference on Telecommunications and Signal Processing. Budapest (Hungary), 2011, p. 500-504. DOI: 10.1109/TSP.2011.6043680

[13] DE LAMARE, R.C., SAMPAIO-NETO, R. Low-complexity variable step-size mechanisms for stochastic gradient algorithms in minimum variance CDMA receivers. IEEE Transactions on Signal Processing, 2006, vol. 54, no. 6, p. 2302-2317. DOI: 10.1109/TSP.2006.873651

[14] WANG, L., CAI, Y., DE LAMARE, R. C. Low-complexity adaptive step size constrained constant modulus SG-based algorithms for blind adaptive beamforming. In Proceedings of the IEEE International Conference on Acoustics, Speech and Signal Processing. Las Vegas (NV, USA), 2008, p. 2593-2596. DOI: 10.1109/ICASSP.2008.4518179

[15] CAI, Y., DE LAMARE, R. C. Low-complexity variable step-size mechanism for code-constrained constant modulus stochastic gradient algorithms applied to CDMA interference suppression. IEEE Transactions on Signal Processing, 2009, vol. 57, no. 1, p. 313-323. DOI: 10.1109/TSP.2008.2005861
[16] DIMITRIJEVIĆ, B. R., MILOŠEVIĆ, N., MARŠÁLEK, R., NIKOLIĆ, Z. B. BPSK receiver based on recursive adaptive filter with remodulation. Radioengineering, vol. 20, no. 4, 2011, p. 932 to 936 , ISSN: $1210-2512$.

[17] DIMITRIJEVIĆ, B. R., NIKOLIĆ, Z. B., MILOŠEVIĆ, N. Performance improvement of MDPSK signal reception in the presence of carrier frequency offset. IEEE Transactions on Vehicular Technology, vol. 61, no. 1, 2012, p. 381-385. DOI: 10.1109/TVT.2011.2177105

[18] HAYKIN, S., MOHER, M. Modern Wireless Communications. New Jersey: Pearson Education, Inc., 2005. ISBN:0-13-124647-6

[19] NWALOZIE, C., OKOROGU, N., UMEH, C., ORAETUE, D. Performance analysis of constant modulus algorithm (CMA) blind adaptive algorithm for smart antennas in a W-CDMA network. International Journal of Engineering Science and Innovative Technology, 2012, vol. 1, no. 2, p. 246-254. ISSN: 2319-5967.

[20] SLOCK, D. T. M. On the convergence behavior of the LMS and the normalized LMS algorithms. IEEE Transactions on Signal Processing, 1993, vol. 41, no. 9, p. 2811-2825. DOI: $10.1109 / 78.236504$

[21] DOUGLAS, S. C. A family of normalized LMS algorithms. IEEE Signal Processing Letters, 1994, vol. 1, no. 3, p. 49-51. DOI: $10.1109 / 97.295321$

\section{About the Authors ...}

Bojan DIMITRIJEVIĆ was born in Leskovac, Serbia, in 1972. He received the B.E.E., M.Sc., and Ph.D. degrees from the University of Niš in 1998, 2002, and 2006, respectively. His research interests include digital signal processing in telecommunications with special focus on interference suppression, adaptive filtering and synchronization, numerical methods in electromagnetics with special focus on FDTD method and signal, material and component modeling.

Vladimir KRSTIĆ was born in Niš, Serbia in 1953. He received the B.E.E., M.Sc., and Ph.D. degrees at the Faculty of Electrical Engineering, University of Belgrade, in 1978, 1990, and 2009, respectively. He is currently the project fellow at the Institute "Mihajlo Pupin" in Belgrade. His research interests are in adaptive equalization and channel identification theory as well as digital signal processing in communications.

Bojana NIKOLIĆ was born in Niš, Serbia in 1982. She received the Dipl. Ing. and Ph.D. degrees in Telecommunications from the Faculty of Electronic Engineering in Niš in 2007 and 2012, respectively. Her field of interest includes wireless communications, digital communications over fading channels and FDTD numerical modeling in electromagnetics. 